\title{
Ictericia obstructiva secundaria a migración de fragmentos de hepatocarcinoma a la vía biliar*
}

\author{
Drs. JUAN HEPP K. ${ }^{1}$, PAULINA BALBONTÍN M. ${ }^{2}$, RODOLFO ARMAS M. ${ }^{2}$, \\ CLAUDIO NAVARRETE G. ${ }^{2}$, HORACIO RÍOS R. ${ }^{1}$, ROBERTO HUMERES A. ${ }^{1}$, \\ GABRIELA RODRÍGUEZ M. ${ }^{1}$, IVÁN ROA E. ${ }^{3}$ \\ Departamento de Cirugía. \\ Departamento de Enfermedades Digestivas. \\ 3 Departamento de Anatomía Patológica. \\ Clínica Alemana, Santiago, Chile.
}

\begin{abstract}
Obstructive jaundice secondary to hepatocellular carcinoma fragments migrated to common bile duct

Obstructive jaundice is a rare presentation of hepatocellular carcinoma (HC), and when it occurs, usually is due to progressive damage from cirrhosis, or extensive tumor infiltration. Tumor growth through the bile duct is being described with increasing frequency as a cause of obstructive jaundice. Rarely, it may be hepatocarcinoma fragments that migrate to the bile duct, obstructing it. We present a case of obstructive jaundice due to migration of fragments of hepatocellular carcinoma to the bile duct in a patient treated 7 years before, for an $\mathrm{HC}$ with a curative resection.
\end{abstract}

Key words: Hepatocellular carcinoma, obstructive jaundice, bile ducts.

\section{Resumen}

La ictericia obstructiva es una presentación poco común en un hepatocarcinoma (HC). Cuando en estos casos existe ictericia, habitualmente se debe a daño progresivo por cirrosis, o a infiltración tumoral extensa. El crecimiento o vaciamiento tumoral hacia la vía biliar se ha descrito ocasionalmente como causa de ictericia obstructiva. En raras ocasiones, puede tratarse de fragmentos de hepatocarcinoma que migran hacia la vía biliar, obstruyéndola. Presentamos un caso de ictericia obstructiva por migración de fragmentos de hepatocarcinoma a la vía biliar, en un paciente tratado 7 años antes por un $\mathrm{HC}$, con resección curativa.

Palabras clave: Hepatocarcinoma, ictericia obstructiva, vía biliar.

\footnotetext{
*Recibido el 26 de Noviembre de 2009 y aceptado para publicación el 12 de Marzo de 2010.

Correspondencia: Dr. Juan Hepp K.

Manquehue Norte 1410, Santiago, Chile. Código Postal: 765-0568.

E-mail: jhepp@alemana.cl
} 


\section{Introducción}

El hepatocarcinoma es la quinta neoplasia más frecuente en el mundo y la tercera causa de muerte por cáncer ${ }^{1}$. Se estima una incidencia anual de 3-5\% en pacientes cirróticos en el mundo, y en Chile una incidencia menor a $9 / 100.000^{2}$.

En estos pacientes, la ictericia aparece en sus etapas finales y en la gran mayoría de los casos se debe al deterioro hepático progresivo debido a la cirrosis, o bien a la infiltración tumoral extensa del parénquima $^{3-5}$. La ictericia obstructiva como presentación inicial es rara y puede deberse a trombos tumorales, hemobilia, infiltración tumoral o compresión tumoral de la vía biliar ${ }^{4,5}$. Poco se ha escrito sobre la migración de fragmentos del tumor hacia la vía biliar como causa de ictericia obstructiva ${ }^{5}$.

El objetivo de este trabajo es presentar un caso clínico en el que se demostró presencia de fragmentos tumorales de hepatocarcinoma en la vía biliar.

\section{Caso clínico}

Varón de 86 años de edad, con antecedentes de Diabetes Mellitus tipo 2 en tratamiento y discreto daño hepático crónico, sin antecedentes categóricos de daño por alcohol, ni elementos de hemocromatosis o de infección por virus de hepatitis B o C. En Noviembre de 2002 se operó por un hepatocarcinoma de $2,5 \mathrm{~cm}$ del segmento hepático 6 , realizándose una resección hepática segmentaria derecha curativa, con márgenes amplios. La determinación de alfa fetoproteína sérica siempre fue negativa. El paciente evoluciona favorablemente sin contratiempos, con controles periódicos de imágenes y exámenes de laboratorio, en los que destaca una bilirrubina levemente elevada $(1,5 \mathrm{mg} / \mathrm{dl})$ de predominio indirecta.

En Enero de 2009, con 86 años de edad, autovalente y con muy buen estado general, presenta alteración de las pruebas hepáticas, con bilirrubina de $1,5 \mathrm{mg} / \mathrm{dl}$, gamaglutamil transferasa (GGT) de 800 U/L (previa 400) y transaminasa GPT-ALT (GPT) de $62 \mathrm{U} / \mathrm{L}$ (previa 33). Se realizó una tomografía computada (TC) de abdomen que muestra una imagen nodular mal definida en la región central del hígado, craneal al segmento proximal de la rama derecha de la vena porta y que determina dilatación de la vía biliar intrahepática hacia el lóbulo izquierdo (Figura 1). La Colangiografía Resonancia Magnética (CRM) solicitada fue diferida por el paciente.

Consulta 8 meses después, con ictericia progresiva, coluria, acolia y baja de peso. Se constata una bilirrubina de $10 \mathrm{mg} / \mathrm{dl}$, plaquetas de $90.000 / \mathrm{mm}^{3}$, y fosfatasas alcalinas (FA) y GGT en aumento. La CRM muestra un nódulo mal definido en el hilio hepático, que rechaza y comprime la vía biliar, determinando dilatación de conductos intrahepáticos (Figura 2). Se plantea una recidiva de su carcinoma hepático o una adenopatía a este nivel. El paciente se encontraba en buenas condiciones generales, autovalente, lúcido y sin estigmas de insuficiencia hepática clínica.

Se decide realizar exploración quirúrgica de la lesión, comprobando la existencia de un hígado con aspecto cirrótico. Se explora la vía biliar, extrayendo coágulos y material gelatinoso, grisáceo, que obstruía la vía biliar. La biopsia rápida de ese material es informado compatible con hepatocarcinoma, lo cual se confirma con la biopsia diferida (Figura 3). Se colocaron 2 prótesis de $7 \mathrm{Fr}$ a hepáticos derecho e izquierdo, además de una cisticostomía descompresora. La bilirrubina cae de 15 a $9 \mathrm{mg} / \mathrm{dl}$, cede la coluria y la acolia. Se consideró que no era de resolución quirúrgica resectiva, como tampoco candidato a trasplante hepático.

Dos semanas después de la cirugía se efectúa una Colangiografía Endoscópica Retrógrada (CER) de control y para re posicionar las prótesis. Se retiran ambas prótesis y se coloca una nueva de $10 \mathrm{Fr}$ al conducto hepático derecho, no logrando canular con catéter el hepático izquierdo (Figura 3), donde existe mayor infiltración tumoral.

Se decide manejo conservador, considerando el daño de base del hígado, la resección hepática previa y la edad del paciente. Se mantendrá con reevaluaciones endoscópicas de su drenaje con endoprótesis según los síntomas. Eventualmente se

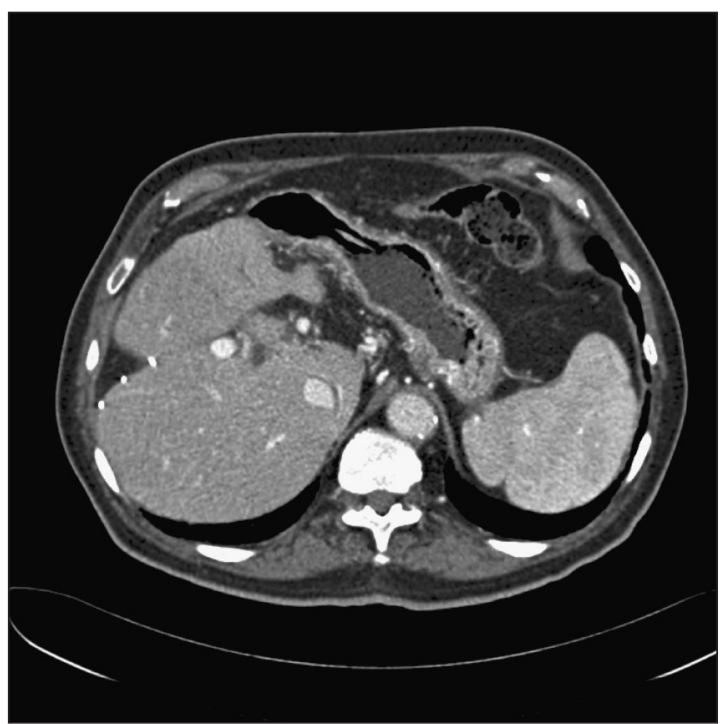

Figura 1. TC abdomen muestra imagen nodular mal definida hepática. 

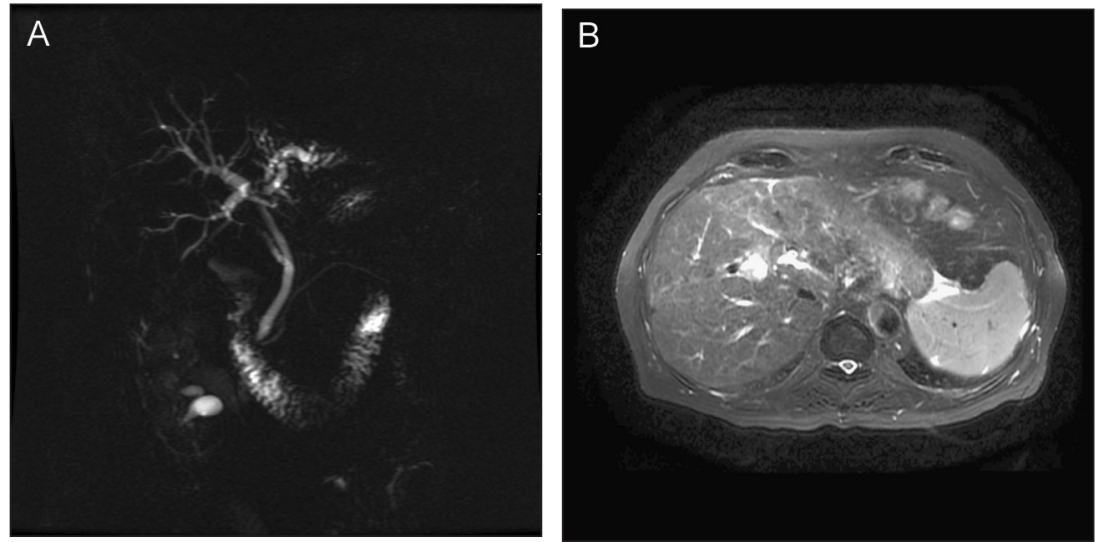

Figura 2. A: Colangio RM: obstrucción a nivel de confluencia de hepáticos y dilatación de vía biliar intrahepática. B: Imagen nodular en hilio hepático.
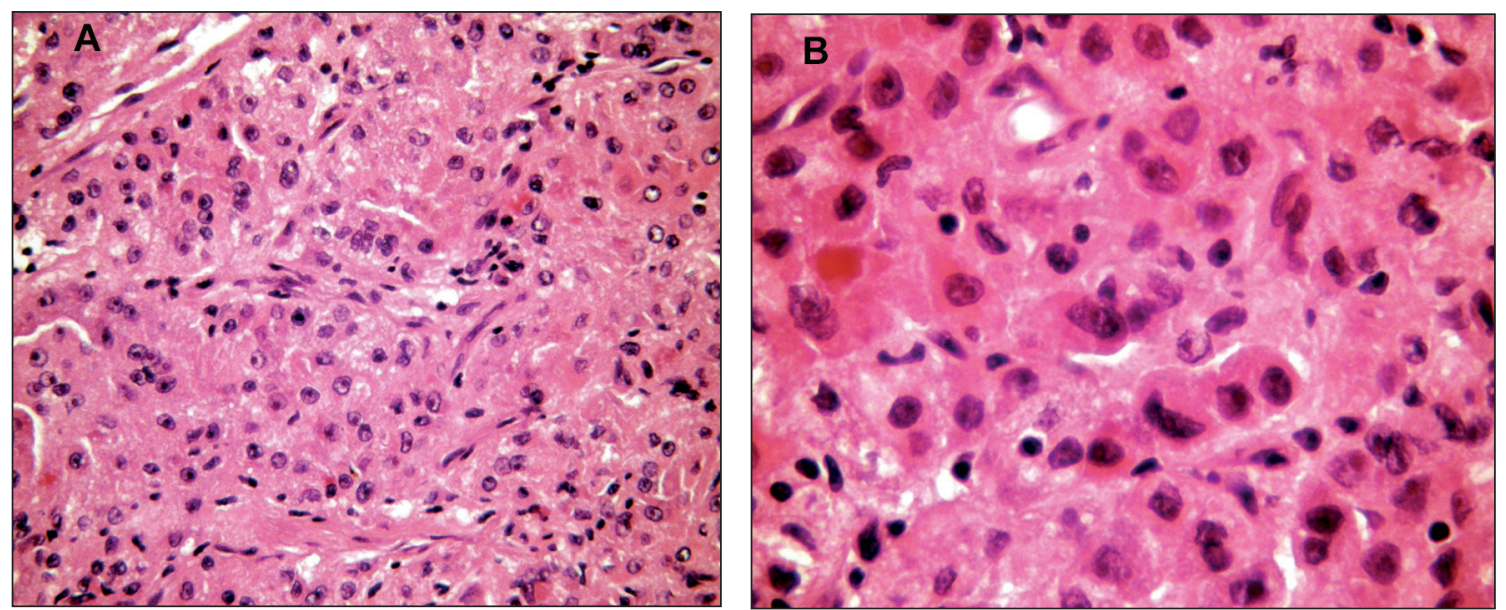

Figura 3. Anatomía Patológica. A: Tumor constituido por proliferación de brotes sólidos y en áreas se reconoce patrón trabecular, formado por células hepáticas con abundante citoplasma eosinófilo, signos degenerativos y núcleos irregulares. No se observan espacios portas ni estructuras lobulillares (HE x 100). B: Grupos celulares que se disponen en trabéculas incompletas con pérdida de la polaridad, citoplasma abundante y núcleos hipercromáticos, con marcadas irregularidades, polimorfismo y anisocariosis (Hex 400).
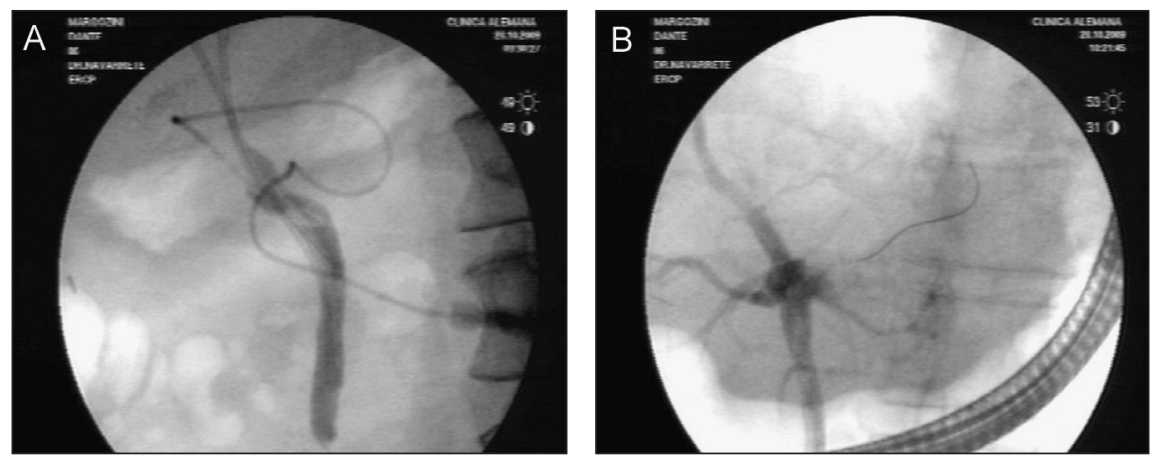

Figura 4. A: Colangiografía transcística muestra prótesis desplazadas, sin salida a duodeno. B: Colangiografía Endoscópica Retrógrada mostrando prótesis en vía biliar derecha y avance sólo de guía a la izquierda. 
realizará drenaje percutáneo de la vía biliar izquierda de considerarlo necesario.

El paciente evoluciona en forma satisfactoria y a las 5 semanas de operado hace su vida activa habitual, reduciendo la GGT y FA, con bilirrubina de $3,5 \mathrm{mg} / \mathrm{dl}$.

\section{Discusión}

La ictericia obstructiva es rara como presentación inicial del hepatocarcinoma, y cuando ocurre, corresponde al llamado "icteric type hepatoma" descrito inicialmente por Lin en $1975^{6}$. El diagnóstico preoperatorio es difícil, y suele confundirse con coledocolitiasis o colangiocarcinoma. La determinación de alfa fetoproteína preoperatoria podría ser de utilidad en el diagnóstico. Los signos más frecuentes son pérdida de peso, náuseas y vómitos, hepatomegalia y aumento importante de bilirrubina y moderado de FA y GGT ${ }^{3,5}$. En este paciente, la historia, los signos clínicos y el laboratorio orientaban al diagnóstico, a pesar de la negatividad de la alfa fetoproteína, e imágenes no concluyentes.

La invasión intrahepática del hepatocarcinoma puede producir ictericia obstructiva por tres causas: (1) crecimiento tumoral hacia la vía biliar intra o extrahepática, (2) fragmentos de tumor necrótico que migran hacia distal, y (3) hemorragia tumoral que finalmente obstruye la vía biliar con coágulos ${ }^{5}$. Estas dos últimas podrían explicar la ictericia de nuestro paciente.

El tratamiento de este tipo de hepatocarcinoma depende de la etapificación del tumor y de la patología hepática subyacente, y puede ser desde la colocación de prótesis por vía endoscópica o percutánea, hasta el trasplante hepático. La resección quirúrgica, es la única que ha demostrado ser curativa y por lo tanto, tiene las mejores tasas de sobrevida ${ }^{3,5,7}$. En el paciente descrito se decidió el manejo sintomático paliativo por tratarse de un segundo hepatocarcinoma en un hígado con daño crónico, en un paciente añoso.

En la literatura están descritos casos de migración de fragmentos de hepatocarcinoma, con una baja frecuencia, siendo mayor en Taiwan y Hong Kong, donde tienen una mayor incidencia de cirrosis y $\mathrm{HC}^{5,8-10}$. También se han publicado casos en India, Inglaterra y Estados Unidos ${ }^{11-13}$. A diferencia de los casos publicados por $\mathrm{Chen}^{5}$, quienes manifestaron episodios de dolor, el paciente comentado tuvo una ictericia progresiva y silenciosa.

Se han descrito además, casos de migración de fragmentos tumorales a la vía biliar distal de otros tipos de cáncer, por ejemplo, cáncer de vesícula, colangiocarcinoma o metástasis de cáncer de colon ${ }^{14}$.
A pesar de tratarse de una causa poco frecuente de ictericia obstructiva, el hepatocarcinoma debe ser considerado en el diagnóstico diferencial de ésta, principalmente en pacientes cirróticos. La presencia de ictericia no siempre es sinónimo de enfermedad avanzada y en algunos casos, pueden beneficiarse de una resección quirúrgica.

\section{Agradecimientos}

A los Drs. Pablo Soffia y Giancarlo Schiapaccasse por su aporte de las imágenes radiológicas.

\section{Referencias}

1. Parkin DM, Bray F, Ferlay J, Pisani P. Estimating The World Cancer Burden: GLOBOCAN 2000. Int J Cancer 2001; 94: 153-156.

2. Bosch X, Ribes J, Díaz M, Cléries R. Primary liver cancer: Worldwide incidence and trends. Gastroenterology 2004; 127: S5-S16.

3. Qin LX, Tang ZY. Hepatocellular carcinoma with obstructive jaundice: diagnosis, treatment and prognosis. World J Gastroenterol 2003; 9: 385-391.

4. Chen MF. Icteric type hepatocellular carcinoma: clinical features, diagnosis and treatment. Chang Gung Med J 2002; 25: 496-501.

5. Chen MF, Jan YY, Jeng LB, Hwang TL, Wang CS, Chen SC. Obstructive jaundice secondary to ruptured hepatocellular carcinoma into the common bile duct. Surgical experiences of 20 cases. Cancer 1994; 1; 73: 1335-1340.

6. Lin TY, Chen KM, Chen YR, Lin WS, Wang TH, Sung JL. Icteric type hepatoma. Med Chir Dig 1975; 4: 267 270.

7. Wang HJ, Kim JH, Kim JH, Kim WH, Kim MW. Hepatocellular carcinoma with tumor thrombi in the bile duct. Hepatogastroenterology 1999; 46: 2495-2499.

8. Lau WY, Mok SD, Leung JW, Li AK. Migrated tumour fragments in common bile ducts from hepatocellular carcinoma. Aust N Z J Surg 1990; 60: 995-997.

9. Lau WY, Leung JW, Li AK. Management of hepatocellular carcinoma presenting as obstructive jaundice. Am J Surg 1990; 160: 280-282.

10. Lai ST, Lam KT, Lee KC. Biliary tract invasion and obstruction by hepatocellular carcinoma: report of five cases. Postgrad Med J 1992; 68: 961-966.

11. Kiev J, Dyslin DC, Vitenas P Jr, Kerstein MD. Obstructive jaundice caused by hepatoma fragments in the common hepatic duct. J Clin Gastroenterol 1990; 12 : 207-213.

12. Sastry RA, Raju GS, Kumar YR. Icterus due to tumor emboli- a rare presentation of hepatoma. Indian J Gastroenterol 1996; 15: 24-25.

13. Kirk JM, Skipper D, Joseph AE, Knee G, Grundy A. 
Intraluminal bile duct hepatocellular carcinoma. Clin Radiol 1994; 49: 886-888.

14. Prinz RA, Ko TC, Maltz SB, Reynes CJ, Marsan RE, Freeark RJ. Common bile duct obstruction by free floating tumor. HPB Surg 1993; 6: 319-323.

15. Hu J, Pi Z, Yu MY, Li Y, Xiong S. Obstructive jaundice caused by tumor emboli from hepatocellular carcinoma. Am Surg1999; 65: 406-410.

16. De Raffele E, Mirarchi M, Bassi F, Cola B. Hepatocellular carcinoma with neoplastic thrombosis of the common hepatic duct. Chir Ital 2008; 60: 849-862.

17. Lau WY, Leow CK, Leung KL, Leung TW, Chan M, Yu SC. Cholangiographic features in the diagnosis and management of obstructive icteric type hepatocellular carcinoma. HPB Surg 2000; 11: 299-306.

18. Cleland PG, Adjukiewicz A. Hepatoma and obstructive jaundice. Postgrad Med J 1980; 56: 371-372.

19. Rhoe BS, Kim H, Jin SY, Jang WI. Hepatoma presen- ting as extrahepatic biliary obstruction due to hemobilia Yonsei Med J 1989; 30: 383-386.

20. Lau W, Leung K, Leung TW, Liew CT, Chan MS, Yu $\mathrm{SC}$, et al. A logical approach to hepatocellular carcinoma presenting with jaundice. Ann Surg 1997; 225: 281-285.

21. Peng BG, Liang LJ, Li SQ, Zhou F, Hua YP, Luo SM. Surgical treatment of hepatocellular carcinoma with bile duct tumor thrombi. World J Gastroenterol 2005; 11: 3966-3969.

22. Nonomura A, Ohta G, Kanai M, Kobayashi K. Hepatocellular carcinoma presenting extrahepatic biliary obstruction. Acta Pathol Jpn 1983; 33: 789-806.

23. Kobayashi N, Kirikoshi H, Higurashi T, Lida H, Mawatari $\mathrm{H}$, Endo H, et al. Tumor fragment impacted at the major duodenal papilla causing obstructive jaundice in a patient with hepatocellular carcinoma. Gastrointest Endosc 2008; 68: 999-1000. 